Research Article

\title{
Multiobjective Optimization Design of Time-Modulated Concentric Circular Ring Arrays
}

\author{
Weilong Liang, ${ }^{1}$ Zhao Wu $\mathbb{D}^{2}{ }^{2}$ and Li Zhang ${ }^{3}$ \\ ${ }^{1}$ Nanjing Research Institute of Electronics Technology, Nanjing 210013, China \\ ${ }^{2}$ College of Physical Science and Technology, Yulin Normal University, Yulin 537000, China \\ ${ }^{3}$ School of Electronic Engineering, Xidian University, Xi'an 710071, China \\ Correspondence should be addressed to Zhao Wu; kianty@163.com
}

Received 16 October 2017; Revised 3 January 2018; Accepted 29 January 2018; Published 25 March 2018

Academic Editor: Jaume Anguera

Copyright ( 2018 Weilong Liang et al. This is an open access article distributed under the Creative Commons Attribution License, which permits unrestricted use, distribution, and reproduction in any medium, provided the original work is properly cited.

A multiobjective approach based on the third evolution step of generalized differential evolution (GDE3) algorithm is proposed for optimizing the time-modulated array (TMA) in this paper. Different from the single-objective optimization, which optimizes a weighted sum of the peak sidelobe level (PSLL) and the peak sideband level (PSBL) of the array, the multiobjective algorithm treats the PSLL and the PSBL as two distinct objectives that are to be optimized simultaneously. Furthermore, not only one outstanding optimization result can be acquired but also a set of solutions known as Pareto front is obtained by using the GDE3 algorithm, which will guide the design of time-modulated array more effectively. Users can choose one appropriate outcome which has a suitable tradeoff between the PSLL and the PSBL. This approach is illustrated through a time-modulated concentric circular ring array (CCRA). The optimal parameters and the corresponding radiation patterns are presented at last. Experimental results reveal that the multiobjective optimization can be an effective approach for the TMA synthesis problems.

\section{Introduction}

The time-modulated array (TMA) was proposed in 1959 by Shanks and Bickmore [1] and then improved by Kummer et al. in 1963 [2]. Compared with the conventional antenna arrays, an additional high-speed RF switch is connected to each antenna element in the TMA, which introduces the fourth dimension, time, into the design. By controlling the switch-on time interval in a period, the TMA has great flexibility in the control of the aperture excitation which tapers the distribution easily and rapidly. So the realization of low/ ultralow sidelobe level (SLL) antenna array becomes much simpler. However, the TMA has an inherent drawback in that there are many sideband signals spaced at multiples of the modulation frequency, which are usually useless. Generally, the sideband level (SBL) needs to be suppressed in order to reduce the energy loss and interference. As the objective function in the TMA pattern synthesis is highly nonlinear and nondifferentiable with different diverse constraint conditions, various algorithms are adopted to optimize the TMA by adjusting the excitation amplitudes or switch-on time sequence. Approaches based on differential evolution (DE) algorithm in [3-5] were proposed to suppress the SBL in the TMA by rearranging the switch-on time. In [6], a linear array with low SLLs, low SBLs, and uniform excitations simultaneously was obtained based on the direct optimization of the switch-on time sequence via the simple genetic algorithm (SGA). Particle swarm optimization (PSO) was used to minimize the power losses in the TMA by properly modifying the modulation sequence [7]. In [8], a novel hybrid algorithm based on the artificial bee colony ( $\mathrm{ABC}$ ) algorithm and $\mathrm{DE}$ algorithm called $\mathrm{ABC}-\mathrm{DE}$ was used to overcome the drawback of the TMA. Furthermore, a hybrid enhanced PSO and DE (hybrid DPSO/DE) was used to optimize the concentric hexagonal antenna array and concentric circular ring array [9]. Also flower pollination algorithm or enhanced flower pollination algorithm is used in the synthesis of circular array antenna [10] and the linear antenna arrays [11].

As mentioned above, the TMA has been optimized by different kinds of optimization algorithms. In these algorithms, single objective is optimized, which means only one 
best result can be concluded after optimization. However, the electromagnetic optimization objectives are often in conflict with each other, and there may not exist a solution that is the global best one. In fact, there are a set of solutions known as Pareto front or nondominated solutions [12-17]. Therefore, multiobjective evolutionary algorithms have been employed to solve complicated antenna design problems such as the optimal synthesis of linear arrays, planar arrays, or concentric ring arrays [18-22].

In this paper, an approach based on the third evolution step of generalized differential evolution (GDE3) [23] is presented for the TMA optimization. In order to demonstrate the methodology, the time-modulated concentric circular ring array (CCRA) is considered. Two objective functions, the peak sidelobe level (PSLL) and peak sideband level (PSBL) of the time-modulated CCRA, are optimized as a biobjective problem. By optimizing the normalized switchon time sequence, the number of elements, and the ring spacing, an extensive set of solutions is obtained, and users can choose the most suitable one from it. This method shows the relationship between the PSLL and the PSBL of the TMA, which indicates that the PSLL is inversely proportional to the PSBL.

The rest of this paper is organized as follows. Section 2 describes properties of the time-modulated CCRA and the parameters defined the radiating structure. Then, the numerical results after optimization are presented in Section 3. Finally, the conclusion is given in Section 4.

\section{Pattern Synthesis with Time Modulation Technique}

The configuration of $N_{r}$ ring CCRA with ring $n$ having $N_{n}$ equally spaced isotropic elements at a radius of $r_{n}$ is shown in Figure 1. The physical distance between adjacent elements on ring $n$ is constant. In the time-modulated CCRA, all the antenna elements in the same ring have the same weight and are controlled by a same high-speed RF switch. The far-field array factor of the time-modulated CCRA is expressed as

$$
A F=e^{j 2 \pi f_{0} t}\left[1+\sum_{n=1}^{N_{r}} \sum_{m=1}^{N_{n}} U_{n}(t) e^{j k r_{n} \sin \theta \cos \left(\varphi-\varphi_{n m}\right)}\right],
$$

where $f_{0}$ is the center frequency, $\theta$ is the elevation angle with respect to $Z$ axis, $\beta$ is the wavenumber, and $U_{n}$ is the periodic switch-on time sequence function in which antenna elements on ring $n$ are switched on for $\tau_{n}\left(0 \leq \tau_{n} \leq T_{\mathrm{p}}\right)$ in each period $T_{\mathrm{p}} . U_{n}$ is defined as

$$
U_{n}(t)= \begin{cases}1, & 0 \leq t \leq \tau_{n} \\ 0, & \text { otherwise }\end{cases}
$$

By decomposing into Fourier series with different frequency components $(s=0, \pm 1, \ldots, \pm \infty)$, the far-field array factor can be described as

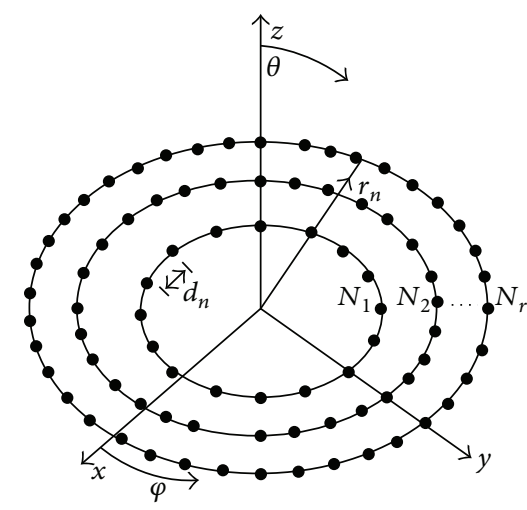

FIgURE 1: The configuration of the CCRA.

$$
A F_{s}=e^{j 2 \pi\left(f_{0}+\left(s / T_{\mathrm{p}}\right)\right) t}\left[1+\sum_{n=1}^{N_{r}} \sum_{m=1}^{N_{n}} \alpha_{s, n} e^{j k r_{n} \sin \theta \cos \left(\varphi-\varphi_{n m}\right)}\right],
$$

where $\alpha_{s, n}$ is the complex amplitude, which is given by

$$
\begin{aligned}
\alpha_{s, n} & =\frac{1}{T_{\mathrm{p}}} \int_{0}^{T_{\mathrm{p}}} U_{n}(t) \cdot e^{-j 2 \pi s\left(t / T_{\mathrm{p}}\right)} \cdot d t \\
& =\frac{\tau_{\mathrm{n}}}{T_{\mathrm{p}}} \cdot \operatorname{sinc}\left(\frac{\pi s \tau_{n}}{T_{\mathrm{p}}}\right) \cdot e^{-j \pi s\left(\tau_{n} / T_{\mathrm{p}}\right)}, \\
\operatorname{sinc}(x) & =\frac{\sin x}{x} .
\end{aligned}
$$

At the center frequency $(s=0)$, the far-field array factor can be simplified as

$$
A F_{0}=e^{j 2 \pi f_{0} t}\left[1+\sum_{n=1}^{N_{r}} \sum_{m=1}^{N_{n}} \frac{\tau_{n}}{T_{\mathrm{p}}} e^{j k r_{n} \sin \theta \cos \left(\varphi-\varphi_{n m}\right)}\right] .
$$

It can be seen from the functions that the far-field array factor of the time-modulated CCRA can be adjusted through controlling the normalized switch-on time sequence $\tau_{n} / T_{\mathrm{p}}$ when all the elements are uniformly excited. Thus, the low PSLL can be realized without complicated feeding network. The PSLL and the PSBL to be minimized can be formulated as the following expressions:

$$
\begin{aligned}
& \operatorname{minimize} \operatorname{PSLL}(G)=20 \log \left[\max _{(\theta, \phi \in Y)} \frac{A F_{0}}{A F_{0 \max }}\right], \\
& \operatorname{minimize} \operatorname{PSBL}(G)=20 \log \left[\max \frac{A F_{1}}{A F_{0 \max }}\right],
\end{aligned}
$$

subject to $\operatorname{FNBW}\left(A F_{0}\right) \leq 30^{\circ}$.

where $Y$ denotes the sidelobe region. $G$ is the optimal variables including the normalized switch-on time sequence $\tau_{n} / T_{\mathrm{p}}$, the ring spacing $r_{n}$, and the number of elements $N_{n}$. The FNBW is the first null beamwidth of the CCRA at the center frequency. It is set as a constraint to maintain the directivity of the array. When the FNBW is larger than $30^{\circ}$, the PSLL and the PSBL are set to large values, thereby eliminating the improper solutions. 


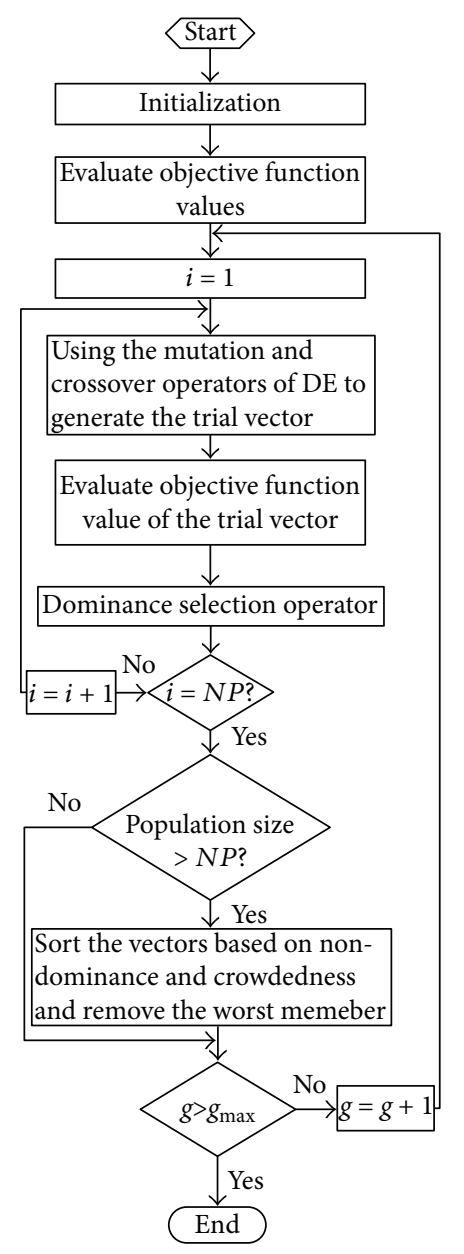

FIgURE 2: The flowchart of the GDE3 algorithm.

To optimize the PSLL of the time-modulated CCRA, and to get the relationship between the PSLL and the PSBL, the multiobjective evolutionary algorithm GDE3 [20] is adopted in this paper. The flowchart of the GDE3 is shown in Figure 2. Without loss of generality, the antenna arrays to be considered in this paper are nine-ring CCRAs and a sixring CCRA with isotropic elements presented in [21]. The spacing between adjacent elements on the same ring is constant and greater than or equal to $\lambda / 2$, where $\lambda$ is the wavelength at the center frequency. The GDE3 algorithm is employed to optimize the normalized switch-on time sequence, the number of elements, and the ring spacing by balancing the PSLL and the PSBL. Parameters for the GDE3 algorithm are set as follows: population size $\mathrm{NP}=100$, scaling factor $F=0.5$, crossover rate $\mathrm{CR}=0.9$, and the maximum number of generation $g_{\max }=2000$. The first design objective is the PSLL, and the second design objective is the PSBL. These two objects are incompatible objective functions. The algorithm is run for 10 times independently for each problem, and the best results are presented in the next section.

\section{Numerical Results}

Consider a uniformly excited nine-ring CCRA presented in [24]. There are nine rings with ring spacing $r_{n}=n \lambda / 2$,
TABLE 1: Ring spacing and numbers of elements for case 1.

\begin{tabular}{lcc}
\hline Ring number & Ring spacing $(\lambda)$ & Number of elements \\
\hline 1 & 0.5 & 6 \\
2 & 1.0 & 12 \\
3 & 1.5 & 18 \\
4 & 2.0 & 25 \\
5 & 2.5 & 31 \\
6 & 3.0 & 37 \\
7 & 3.5 & 43 \\
8 & 4.0 & 50 \\
9 & 4.5 & 56 \\
\hline
\end{tabular}

and spacing between adjacent elements within the same ring $d_{n}=\lambda / 2$. Parameters of the nine-ring CCRA are listed in Table 1.

To realize low PSLL, three examples are presented in this paper. Firstly, the normalized switch-on time sequence is optimized in case 1, with the ring spacing and numbers of elements unchanged.

To reduce the PSLL, a thin nine-ring CCRA is considered as case 2 . The ring spacing remains unchanged like case 1 . The spacing between adjacent elements within the same ring and the normalized switch-on time sequence are optimized simultaneously.

In case 3 , to realize much lower PSLL with acceptable PSBL, the ring spacing, the number of elements, and the normalized switch-on time sequence for each ring are all optimized. For comparison, the six-ring CCRA presented in [21] is considered.

As is known, the Pareto front of biobjective problem offers a set of solutions that fulfill the aforementioned constrain. So in each case, three representative individuals, denoted by A, B, and C, are selected for the design. Individual A has the lowest PSBL, and individual B has the lowest PSLL. In consideration of practical using and comparison, the PSLL of individual $\mathrm{C}$ is as low as possible if the PSBL is close to $-20 \mathrm{~dB}$. The numerical results of three examples are shown in the following.

3.1. Case 1. The Pareto front of case 1 is shown in Figure 3. The circles in the figure represent the performance of each individual. From the picture, it can be seen that the PSLL varies inversely proportional to the PSBL.

The performances and the optimized normalized switch-on time sequences of the three representative individuals are listed in Tables 2 and 3, respectively. Obviously, individual $\mathrm{A}^{1}$ has the lowest PSBL, but its PSLL is also the highest. For individual $\mathrm{B}^{1}$, it has the lowest PSLL but with the highest PSBL. There is a tradeoff between the PSLL and the PSBL for individual $\mathrm{C}^{1}$ with PSLL $=-25.4 \mathrm{~dB}$ and $\mathrm{PSBL}=-20.14 \mathrm{~dB}$. Compared with the conventional nine-ring CCRA in [21], the time-modulated individual $\mathrm{C}^{1}$ has much lower PSLL whilst it has acceptable PSBL, though the FNBW becomes little larger. As is shown in Table 3, to realize low PSLL, the switch-on times of elements in marginal rings are much lower than those of elements in center ring as 


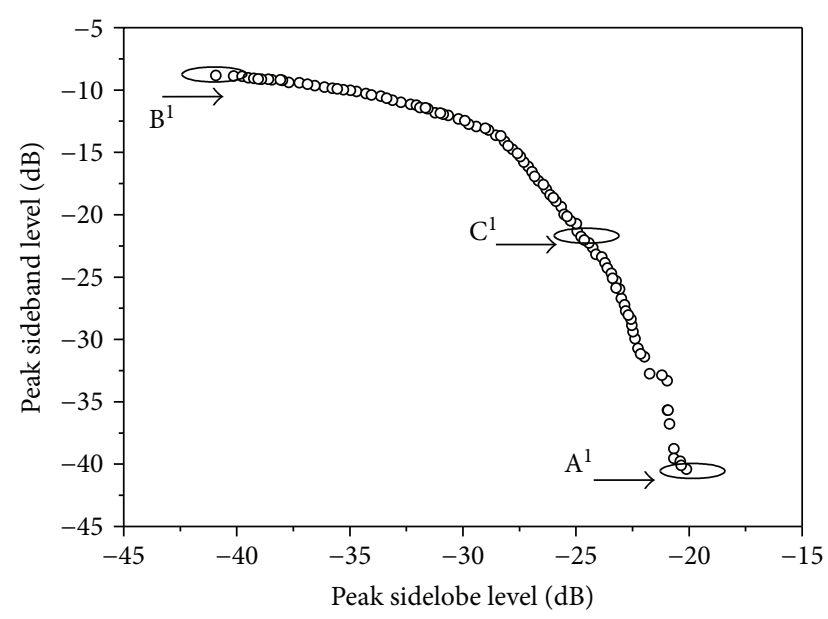

Figure 3: The Pareto front for case 1.

TABle 2: Performances of three individuals in case 1.

\begin{tabular}{lccc}
\hline Individual & PSLL $(\mathrm{dB})$ & PSBL $(\mathrm{dB})$ & FNBW $(\mathrm{deg})$ \\
\hline $\mathrm{A}^{1}$ & -20.11 & -40.42 & 16 \\
$\mathrm{~B}^{1}$ & -40.92 & -8.82 & 25.2 \\
$\mathrm{C}^{1}$ & -25.40 & -20.14 & 20.6 \\
\hline
\end{tabular}

TABLE 3: The normalized switch-on time sequences for individuals $\mathrm{A}^{1}, \mathrm{~B}^{1}$, and $\mathrm{C}^{1}$.

\begin{tabular}{lccc}
\hline Ring number & $\mathrm{A}^{1}$ & $\begin{array}{c}\tau_{n} / T_{\mathrm{p}} \\
\mathrm{B}^{1}\end{array}$ & $\mathrm{C}^{1}$ \\
\hline 1 & 0.9268 & 0.9949 & 0.9956 \\
2 & 0.9456 & 0.9988 & 0.9685 \\
3 & 0.9814 & 0.9545 & 0.9412 \\
4 & 0.9818 & 0.6999 & 0.9977 \\
5 & 0.9673 & 0.5898 & 0.8362 \\
6 & 0.9834 & 0.3792 & 0.1515 \\
7 & 0.9799 & 0.2443 & 0.8213 \\
8 & 0.1007 & 0.1486 & 0.1197 \\
9 & 0.9781 & 0.1005 & 0.1011 \\
\hline
\end{tabular}

individuals $\mathrm{B}^{1}$ and $\mathrm{C}^{1}$ show. It indicates that the tapered normalized switch-on time sequence is equal to the tapered excitation distribution. Meanwhile, to realize low PSBL, the normalized switch-on time sequence should be as large as possible just like $\mathrm{A}^{1}$. The radiation patterns of individual $\mathrm{C}^{1}$ are presented in Figure 4. It can be seen that the PSLL is reduced to $-25.4 \mathrm{~dB}$, which is $8 \mathrm{~dB}$ smaller than that of the conventional uniform CCRA, whilst the optimized CCRA has relatively low PSBL. The numerical results show that the time modulation approach is an achievable method for reducing the PSLL even if the amplitude excitations are uniform. Meanwhile, the multiobjective approach offers different solutions for the tradeoff between the PSLL and the PSBL, and a user can choose an appropriate one for the design.

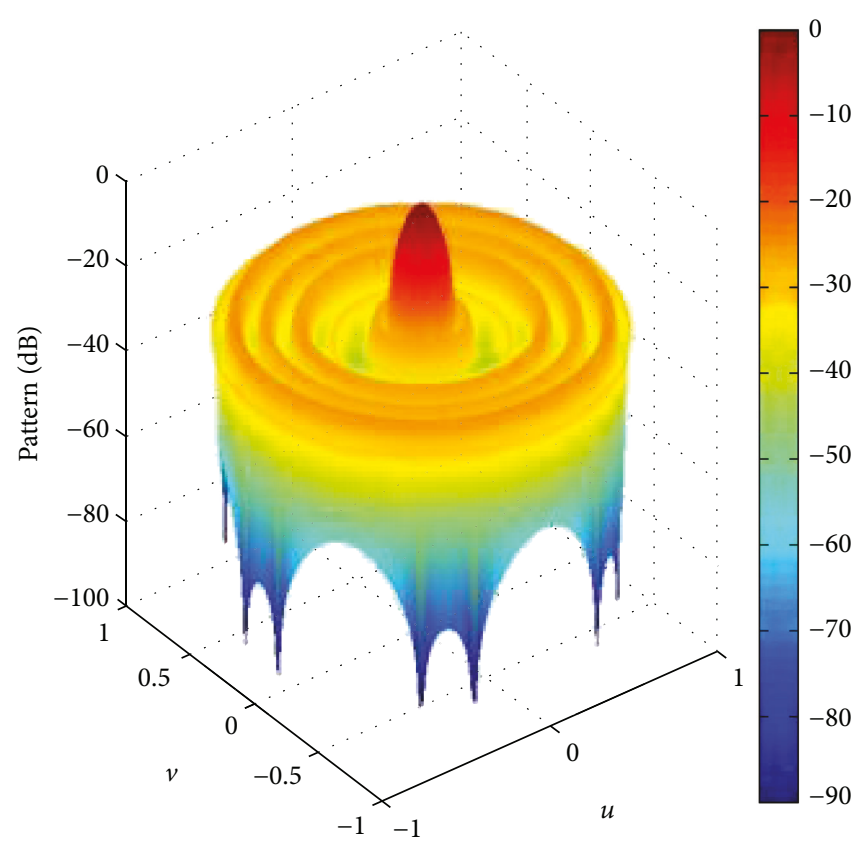

(a)

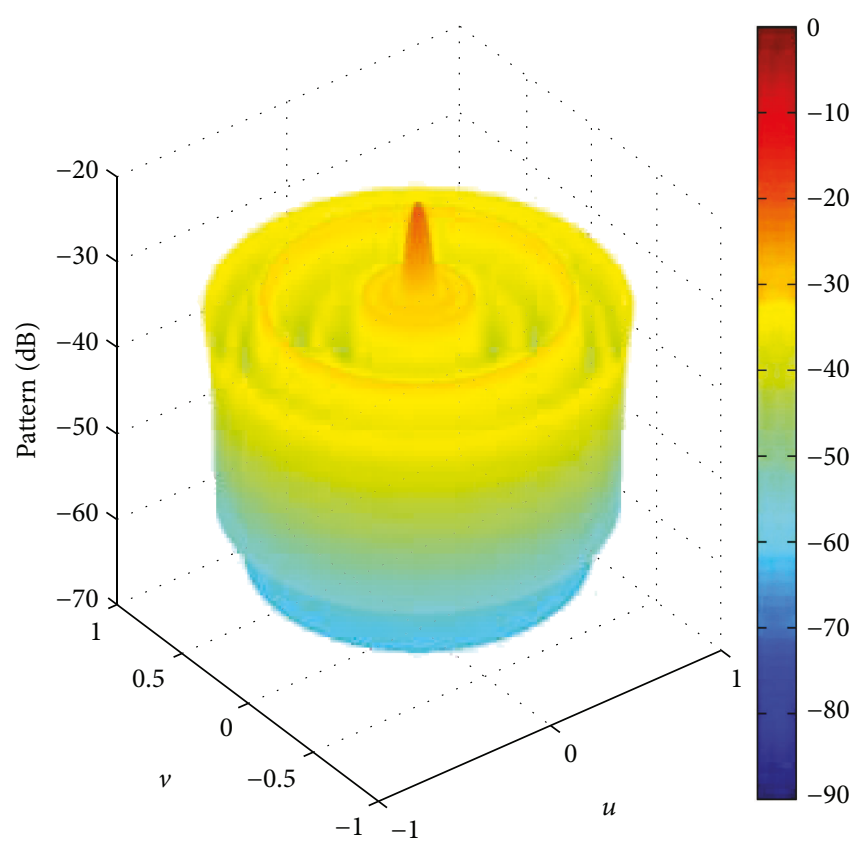

(b)

FIgURE 4: Radiation patterns for individual $C^{1}$ : (a) at the center frequency $(s=0)$ and $(\mathrm{b})$ at the first sideband $(s=1)$.

3.2. Case 2. The Pareto front is shown in Figure 5. The PSLL varies from $-24.34 \mathrm{~dB}$ to $-42.70 \mathrm{~dB}$, and the PSBL varies from $-12.06 \mathrm{~dB}$ to $-40.28 \mathrm{~dB}$. The performances of the marginal individuals $\mathrm{A}^{2}$ and $\mathrm{B}^{2}$ and the acceptable individual $\mathrm{C}^{2}$ are shown in Table 4. It can be seen that individual $\mathrm{C}^{2}$ has a well balance between the PSLL and the PSBL. Compared with that in [21], the time-modulated individual $\mathrm{C}^{2}$ has lower PSLL $=-33.95 \mathrm{~dB}$, which is $8 \mathrm{~dB}$ lower than the conventional CCRA, whilst it has acceptable PSBL $=-20.01 \mathrm{~dB}$. The number of elements and the normalized switch-on time 


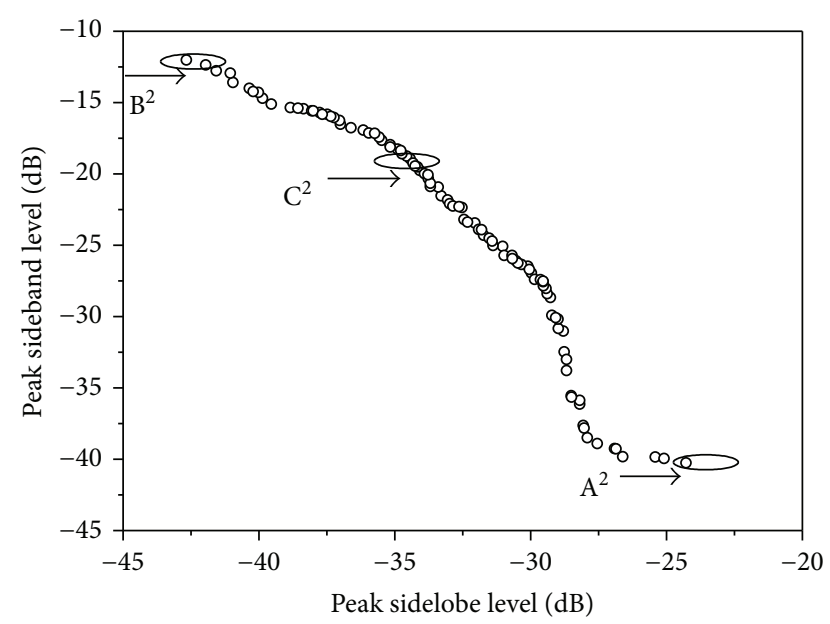

Figure 5: The Pareto front for case 2.

TABLE 4: Performances of three individuals in case 2.

\begin{tabular}{lccc}
\hline Individual & PSLL $(\mathrm{dB})$ & PSBL $(\mathrm{dB})$ & FNBW $(\mathrm{deg})$ \\
\hline $\mathrm{A}^{2}$ & -24.34 & -40.28 & 17.2 \\
$\mathrm{~B}^{2}$ & -42.70 & -12.06 & 26.8 \\
$\mathrm{C}^{2}$ & -33.95 & -20.01 & 22.8 \\
\hline
\end{tabular}

TABLE 5: The numbers of elements and the normalized switch-on time sequences for individuals $\mathrm{A}^{2}, \mathrm{~B}^{2}$, and $\mathrm{C}^{2}$.

\begin{tabular}{lcccccc}
\hline \multirow{2}{*}{ Ring number } & \multicolumn{3}{c}{ Number } & \multicolumn{3}{c}{$\tau_{n} / T_{\mathrm{p}}$} \\
& $\mathrm{A}^{2}$ & $\mathrm{~B}^{2}$ & $\mathrm{C}^{2}$ & $\mathrm{~A}^{2}$ & $\mathrm{~B}^{2}$ & $\mathrm{C}^{2}$ \\
\hline 1 & 6 & 6 & 6 & 0.9806 & 0.9945 & 0.9862 \\
2 & 12 & 12 & 13 & 0.9837 & 0.9740 & 0.9985 \\
3 & 19 & 17 & 18 & 0.9896 & 0.9093 & 0.9849 \\
4 & 25 & 20 & 20 & 0.9917 & 0.8247 & 0.9592 \\
5 & 20 & 21 & 19 & 0.9784 & 0.7642 & 0.8653 \\
6 & 23 & 26 & 22 & 0.9884 & 0.4796 & 0.8162 \\
7 & 25 & 31 & 25 & 0.9961 & 0.2931 & 0.7361 \\
8 & 28 & 43 & 38 & 0.1062 & 0.1430 & 0.1345 \\
9 & 55 & 31 & 45 & 0.9662 & 0.1063 & 0.1366 \\
\hline
\end{tabular}

sequences for individuals $\mathrm{A}^{2}, \mathrm{~B}^{2}$, and $\mathrm{C}^{2}$ are listed in Table 5. It can be concluded that, to realize low PSLL, the key point is the number of elements in marginal rings. After optimization, the number of elements in the first three rings changes little for individuals $\mathrm{A}^{2}, \mathrm{~B}^{2}$, and $\mathrm{C}^{2}$. The total number of elements decreases from 278 in case 1 to 206 in case 2 for individual $\mathrm{C}^{2}$. Also it can be seen that the tapered excitation distribution transforms into the tapered switch-on time sequence in $\mathrm{B}^{2}$ and $\mathrm{C}^{2}$, which reveals that, even with the uniform excitation, the time-modulated technology can realize much lower PSLL with acceptable PSBL than the conventional CCRA can. The multiobjective optimization can also offer the relationship between the PSLL and the PSBL, and users can select one appropriate mode from it. The radiation patterns for individual $\mathrm{C}^{2}$ are shown in Figure 6.

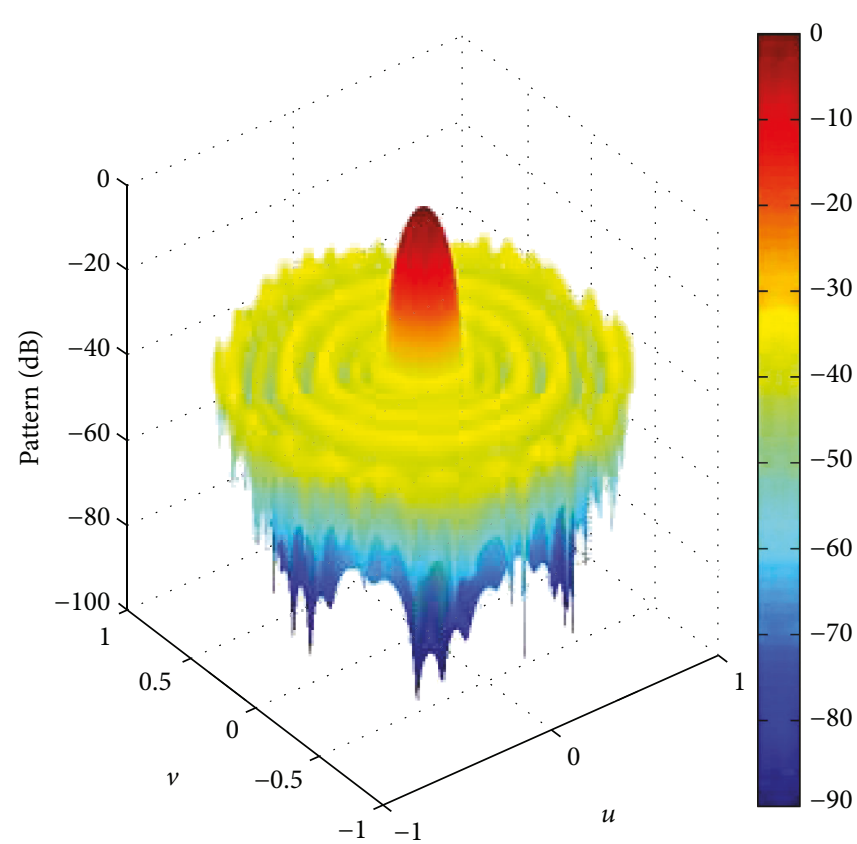

(a)

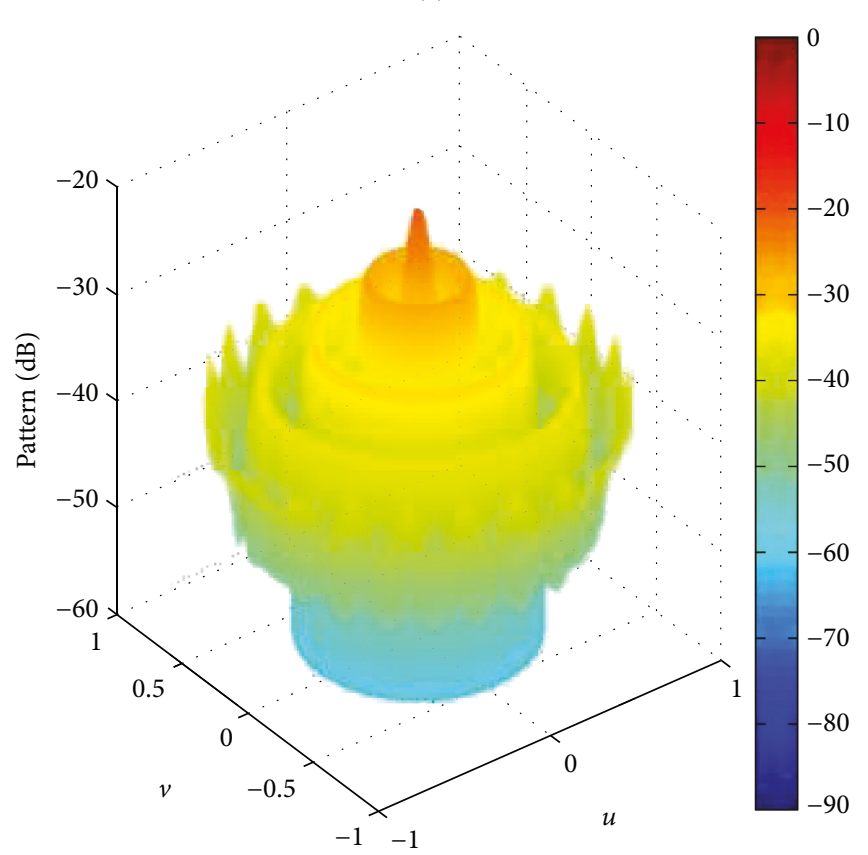

(b)

FIgURE 6: Radiation patterns for individual $C^{2}$ : (a) at the center frequency $(s=0)$ and $(b)$ at the first sideband $(s=1)$.

3.3. Case 3. Figure 7 shows the Pareto front of case 3. From the figure, it can be seen that the Pareto front offers a set of solutions which have the tradeoff between the PSLL and the PSBL. We may find different design parameters for different requirements from the Pareto front.

$\mathrm{A}^{3}, \mathrm{~B}^{3}$, and $\mathrm{C}^{3}$ are chosen as three representative individuals acquired by the GDE3 algorithm. Performances of these three individuals are shown in Table 6. Individual $\mathrm{A}^{3}$ has the lowest PSBL with highest PSLL. Individual $\mathrm{B}^{3}$ has the lowest PSLL but with highest PSBL. For individual $\mathrm{C}^{3}$, there is a 


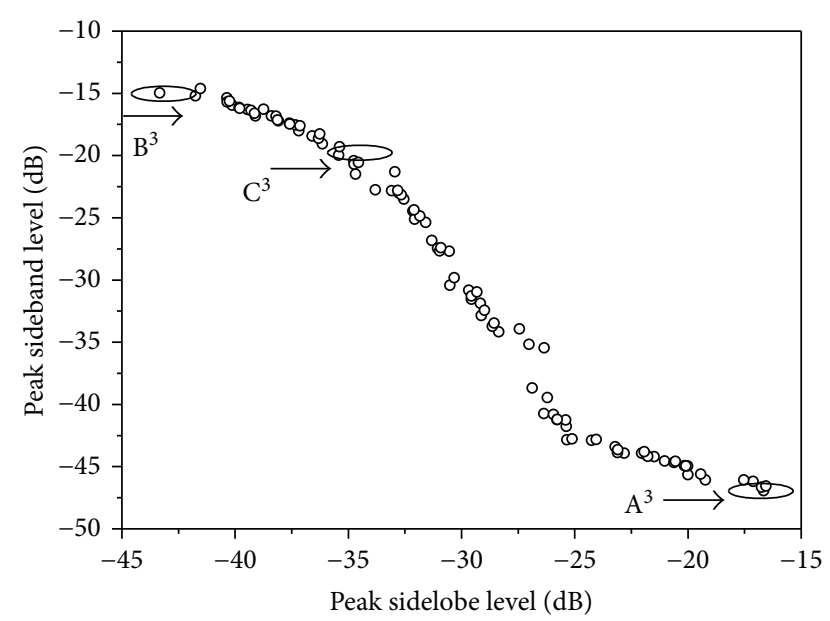

Figure 7: The Pareto front for case 3.

TABLE 6: Performances of three individuals in case 3.

\begin{tabular}{lccc}
\hline Individual & PSLL $(\mathrm{dB})$ & PSBL $(\mathrm{dB})$ & FNBW $(\mathrm{deg})$ \\
\hline $\mathrm{A}^{3}$ & -16.61 & -46.97 & 8.8 \\
$\mathrm{~B}^{3}$ & -43.40 & -14.96 & 28.4 \\
$\mathrm{C}^{3}$ & -34.58 & -20.55 & 24.4 \\
\hline
\end{tabular}

TABLE 7: The ring spacing for individuals $A^{3}, B^{3}$, and $C^{3}$.

\begin{tabular}{lccc}
\hline Ring number & \multicolumn{3}{c}{ Ring spacing $(\lambda)$} \\
\hline 1 & $\mathrm{~A}^{3}$ & $\mathrm{~B}^{3}$ & $\mathrm{C}^{3}$ \\
2 & 0.8999 & 0.5937 & 0.5543 \\
3 & 2.2715 & 1.2132 & 1.1770 \\
4 & 3.4688 & 1.9433 & 1.8761 \\
5 & 4.4764 & 2.7259 & 2.6182 \\
6 & 5.8588 & 3.5232 & 3.3798 \\
\hline
\end{tabular}

TABLE 8: The numbers of elements for individuals $A^{3}, B^{3}$, and $C^{3}$.

\begin{tabular}{lccc}
\hline \multirow{2}{*}{ Ring number } & \multicolumn{3}{c}{$\begin{array}{c}\text { Number of elements } \\
\mathrm{B}^{3}\end{array}$} \\
\hline 1 & 10 & 7 & $\mathrm{C}^{3}$ \\
2 & 18 & 15 & 7 \\
3 & 43 & 21 & 15 \\
4 & 42 & 22 & 22 \\
5 & 66 & 23 & 21 \\
6 & 88 & 46 & 25 \\
\hline
\end{tabular}

tradeoff between the PSLL and the PSBL with PSLL = $-33.7 \mathrm{~dB}$ and PSBL $=-20.24 \mathrm{~dB}$. Meanwhile the PSLL of individual $\mathrm{C}^{3}$ is nearly $6 \mathrm{~dB}$ lower than that of [21]. The optimal ring spacing, number of elements, and normalized switch-on time sequences for individuals $\mathrm{A}^{3}, \mathrm{~B}^{3}$, and $\mathrm{C}^{3}$ are listed in Tables 7-9, respectively. It can be seen that the optimal ring spacing becomes larger than that of case 1 . The distribution relationships for the number of elements and the normalized
TABLE 9: The normalized switch-on time sequences for individuals $\mathrm{A}^{3}, \mathrm{~B}^{3}$, and $\mathrm{C}^{3}$.

\begin{tabular}{lccc}
\hline Ring number & \multicolumn{3}{c}{$\tau_{n} / T_{\mathrm{p}}$} \\
\hline 1 & $\mathrm{~A}^{3}$ & $\mathrm{~B}^{3}$ & $\mathrm{C}^{3}$ \\
2 & 0.9878 & 0.8974 & 0.9755 \\
3 & 0.9776 & 0.9737 & 0.9759 \\
4 & 0.9960 & 0.9348 & 0.9617 \\
5 & 0.9954 & 0.8002 & 0.9479 \\
6 & 0.9965 & 0.3768 & 0.7036 \\
\hline
\end{tabular}

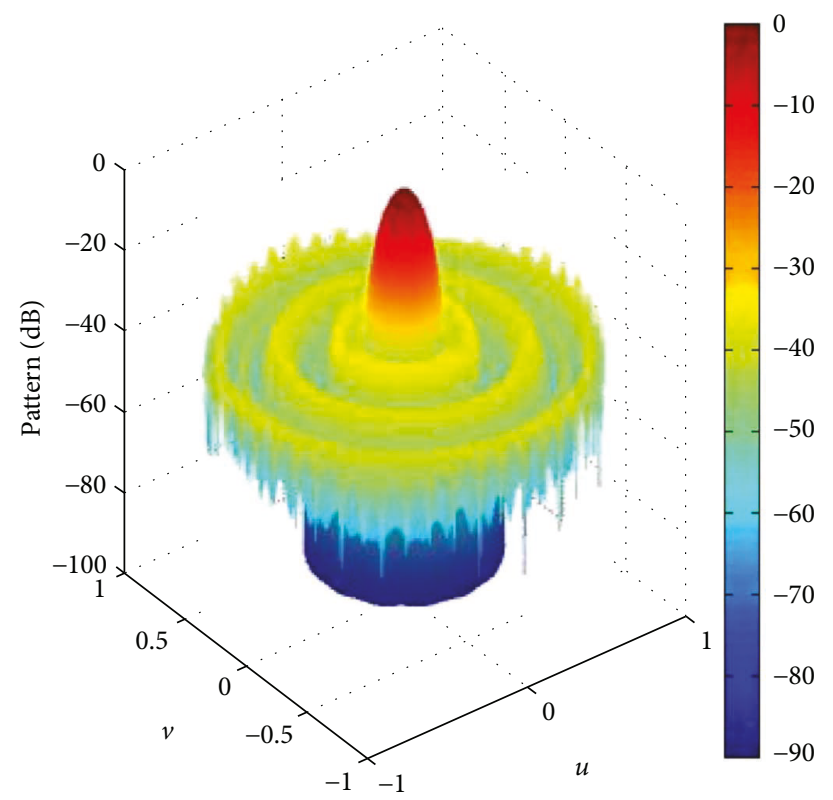

(a)

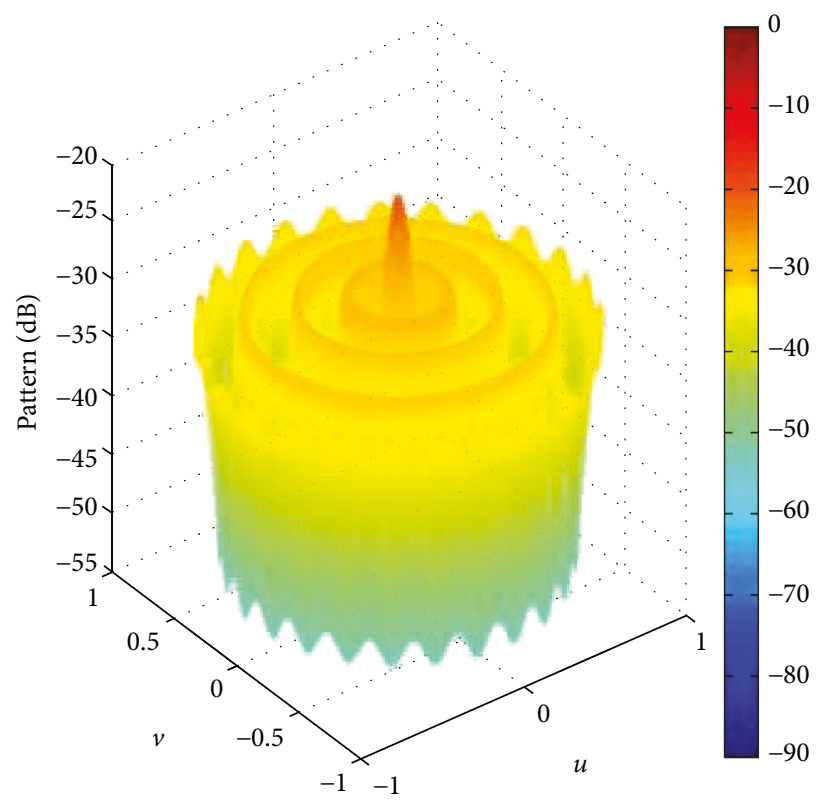

(b)

FIgURE 8: Radiation patterns for individual $C^{3}$ : (a) at the center frequency $(s=0)$ and $(\mathrm{b})$ at the first sideband $(s=1)$. 
switch-on time sequence are the same with case 1 and case 2 . Furthermore, the radiation patterns for individual $C^{3}$ are presented in Figure 8. It can be seen that the optimized individual has both low PSLL and low PSBL.

\section{Conclusion}

In this paper, an attempt based on the multiobjective algorithm is made to solve the pattern synthesis problems of the time-modulated CCRA. By utilizing the GDE3 multiobjective algorithm, a set of solutions known as the Pareto front is obtained, which is beneficial to the design of timemodulated antenna array. In this design, the PSLL and the PSBL are set as two objectives. Three examples are studied in detail. Firstly, the normalized switch-on time sequence is optimized only. Then, both the number of elements and the normalized switch-on time sequence are optimized. The ring spacing, the number of elements within the same ring, and the normalized switch-on time sequence are all optimized at last. Moreover, the corresponding Pareto front, parameters of representative individuals, and radiation patterns are presented for all cases. Compared with the conventional CCRA, the selected three time-modulated CCRAs, $C^{1}$, $\mathrm{C}^{2}$, and $\mathrm{C}^{3}$, have lower PSLL and acceptable PSBL. In addition, users can choose the most appropriate individuals from the Pareto front to fulfill different antenna design requirements. The numerical results reveal that the approach based on the multiobjective optimization is an effective method for the TMA design.

\section{Conflicts of Interest}

The authors declare that there is no conflict of interest regarding the publication of this article.

\section{Acknowledgments}

This work is supported by the Doctoral Scientific Research Foundation of Yulin Normal University G2017002.

\section{References}

[1] H. E. Shanks and R. W. Bickmore, "Four-dimensional electromagnetic radiators," Canadian Journal of Physics, vol. 37, no. 3, pp. 263-275, 1959.

[2] W. Kummer, A. Villeneuve, T. Fong, and F. Terrio, "Ultra-low sidelobes from time-modulated arrays," IEEE Transactions on Antennas and Propagation, vol. 11, no. 6, pp. 633-639, 1963.

[3] S. Yang, Y. B. Gan, and A. Qing, "Sideband suppression in time-modulated linear arrays by the differential evolution algorithm," IEEE Antennas and Wireless Propagation Letters, vol. 1, no. 1, pp. 173-175, 2002.

[4] M. Huang, S. Yang, G. Li, and Z. Nie, "Synthesis of low and equal-ripple sidelobe patterns in time-modulated circular antenna arrays," Journal of Infrared, Millimeter, and Terahertz Waves, vol. 30, no. 8, pp. 802-812, 2009.

[5] L. Zheng, S. Yang, Q. Zhu, and Z. Nie, "Synthesis of pencilbeam patterns with time-modulated concentric circular ring antenna arrays," in PIERS Proceedings, pp. 372-376, Suzhou, China, 2011.
[6] S. Yang, Ye, A. Qing, and P. K. Tan, "Design of a uniform amplitude time modulated linear array with optimized time sequences," IEEE Transactions on Antennas and Propagation, vol. 53, no. 7, pp. 2337-2339, 2005.

[7] L. Poli, P. Rocca, L. Manica, and A. Massa, "Handling sideband radiations in time-modulated arrays through particle swarm optimization," IEEE Transactions on Antennas Propagation, vol. 58, no. 4, pp. 1408-1411, 2010.

[8] J. Yang, W.-T. Li, X.-W. Shi, L. Xin, and J.-F. Yu, "A hybrid ABC-DE algorithm and its application for time-modulated arrays pattern synthesis," IEEE Transactions on Antennas and Propagation, vol. 61, no. 11, pp. 5485-5495, 2013.

[9] M. A. Mangoud, H. M. Elragal, and M. T. Alshara, "Design of time modulated concentric circular and concentric hexagonal antenna array using hybrid enhanced particle swarm optimisation and differential evolution algorithm," IET Microwaves, Antennas \& Propagation, vol. 8, no. 9, pp. 657-665, 2014.

[10] V. S. S. S. Chakravarthy Vedula, S. R. Chowdary Paladuga, and M. Rao Prithvi, "Synthesis of circular array antenna for sidelobe level and aperture size control using flower pollination algorithm," International Journal of Antennas and Propagation, vol. 2015, Article ID 819712, 9 pages, 2015.

[11] U. Singh and R. Salgotra, "Pattern synthesis of linear antenna arrays using enhanced flower pollination algorithm," International Journal of Antennas and Propagation, vol. 2017, Article ID 7158752, 11 pages, 2017.

[12] K. Deb, Multi-Objective Optimization Using Evolutionary Algorithms, John Wiley \& Sons, Chichester, 2001.

[13] C. A. Coello Coello, G. B. Lamont, and D. A. Van Veldhuized, Evolutionary Algorithms for Solving Multi-Objective Problems, Springer, New York, 2007.

[14] R. Storn and K. Price, "Differential evolution-a simple and efficient heuristic for global optimization over continuous spaces," Journal of Global Optimization, vol. 11, no. 4, pp. 341-359, 1997.

[15] R. T. Marler and J. S. Arora, "Survey of multi-objective optimization methods for engineering," Structural and Multidisciplinary Optimization, vol. 26, no. 6, pp. 369-395, 2004.

[16] K. Deb, A. Pratap, S. Agarwal, and T. Meyarivan, "A fast and elitist multiobjective genetic algorithm: NSGA-II," IEEE Transactions on Evolutionary Computation, vol. 6, no. 2, pp. 182-197, 2002.

[17] W. Li, Y. Hei, J. Yang, and X. Shi, “3D pattern synthesis of time-modulated conformal arrays with a multiobjective optimization approach," International Journal of Antennas and Propagation, vol. 2014, Article ID 687124, 12 pages, 2014.

[18] S. Pal, B. Y. Qu, S. Das, and P. N. Suganthan, "Optimal synthesis of linear antenna array with multi-objective differential evolution," Progress In Electromagnetics Research B, vol. 21, pp. 87-111, 2010.

[19] J. S. Petko and D. H. Werner, "Pareto optimization of thinned planar arrays with elliptical mainbeams and low sidelobe levels," IEEE Transactions on Antennas and Propagation, vol. 59, no. 5, pp. 1748-1751, 2011.

[20] D. Bianchi, S. Genovesi, and A. Monorchio, "Constrained Pareto optimization of wide band and steerable concentric ring arrays," IEEE Transactions on Antennas and Propagation, vol. 60, no. 7, pp. 3195-3204, 2012.

[21] S. K. Goudos, K. A. Gotsis, K. Siakavara, E. E. Vafiadis, and J. N. Sahalos, "A multi-objective approach to subarrayed linear antenna arrays design based on memetic differential 
evolution," IEEE Transactions on Antennas and Propagation, vol. 61, no. 6, pp. 3042-3052, 2013.

[22] Z. Li, Y.-C. Jiao, B. Chang, and Z.-B. Weng, "Multiobjective optimization design of concentric ring arrays with $3 \mathrm{D}$ beam scanning using differential evolution algorithm," International Journal of Numerical Modelling: Electronic Networks, Devices and Fields, vol. 26, no. 6, pp. 602-619, 2013.

[23] S. Kukkonen and J. Lanpinen, "GDE3: the third evolution step of generalized differential evolution," in The 2005 IEEE Congress on Evolutionary Computation, vol. 1, pp. 443-450, Edinburgh, Scotland, UK, 2005.

[24] R. L. Haupt, "Optimized element spacing for low sideband concentric ring arrays," IEEE Transactions on Antennas and Propagation, vol. 56, no. 1, pp. 266-268, 2008. 


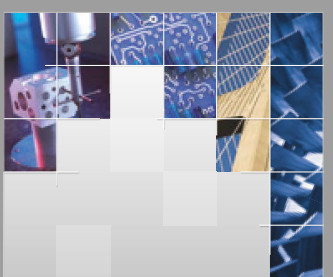

\section{Enfincering}
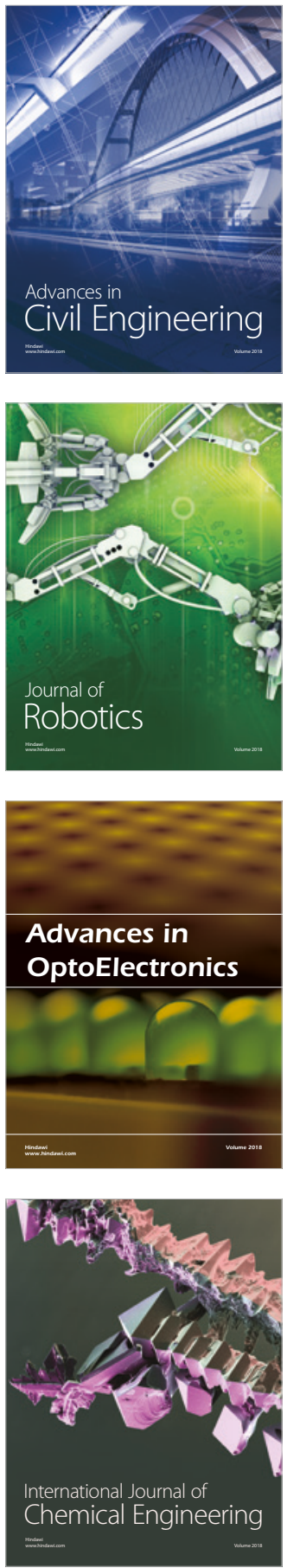

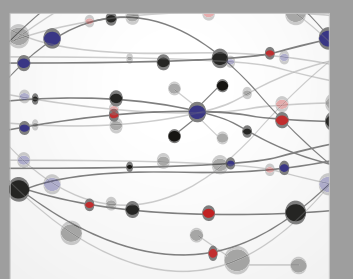

\section{Rotating \\ Machinery}

The Scientific World Journal

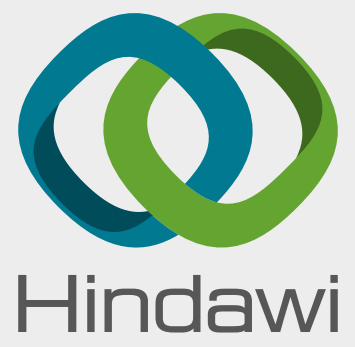

Submit your manuscripts at

www.hindawi.com
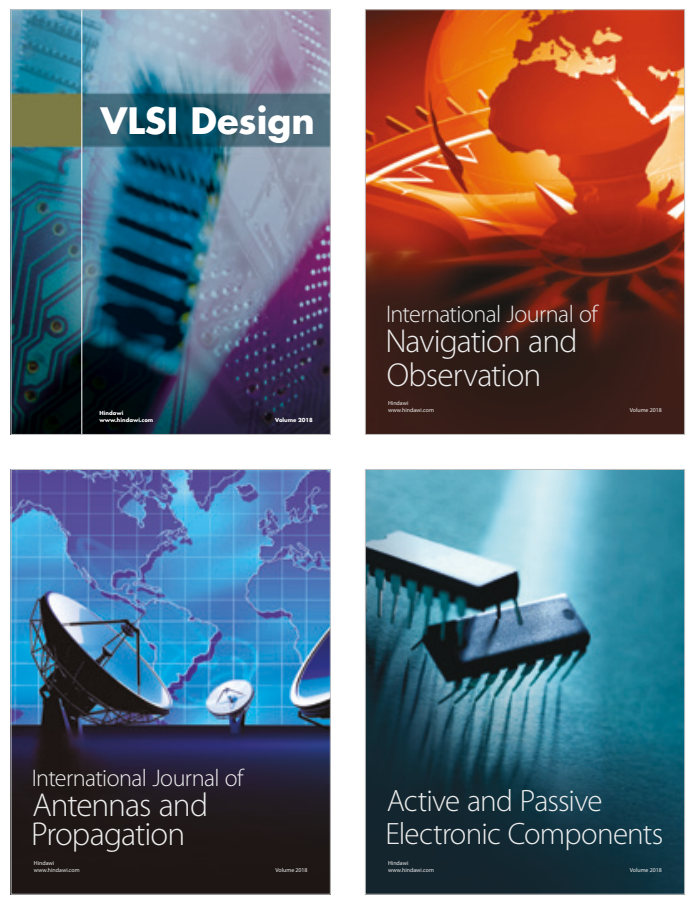
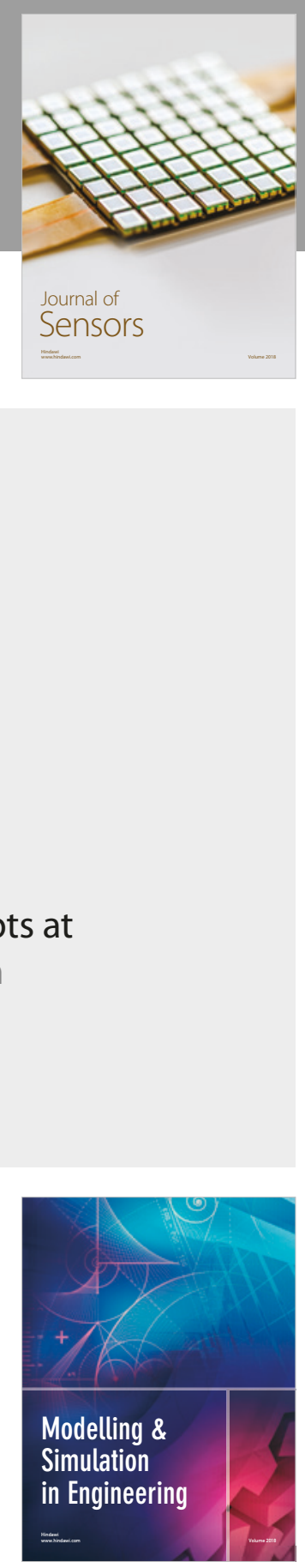

\section{Advances \\ Multimedia}
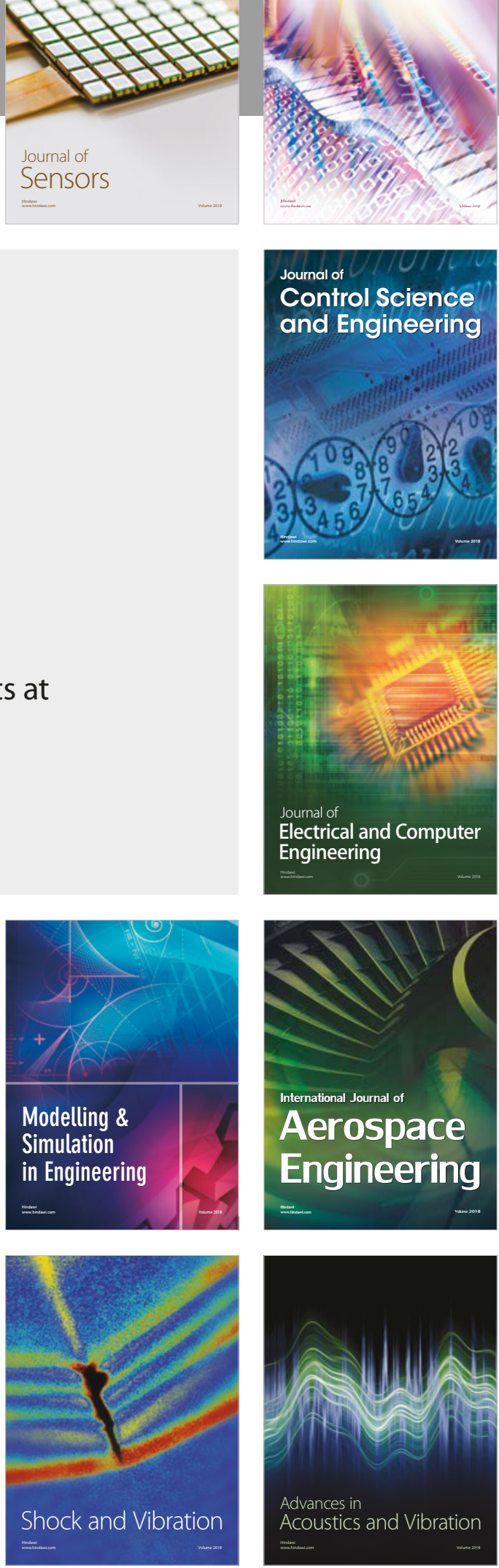